\title{
Scores to Identify Occult Cancer in Venous Thromboembolism: Do They Work?
}

\author{
David Jiménez ${ }^{1}$ Behnood Bikdeli²,3,4
}

\author{
${ }^{1}$ Respiratory Department, Hospital Ramón y Cajal, Universidad de \\ Alcalá (IRYCIS), Madrid, Spain \\ ${ }^{2}$ Division of Cardiology, Department of Medicine, Columbia \\ University Medical Center, New York-Presbyterian Hospital, \\ New York, New York, United States \\ ${ }^{3}$ Center for Outcomes Research and Evaluation (CORE), Yale \\ University School of Medicine, Yale University, New Haven, \\ Connecticut, United States \\ ${ }^{4}$ Cardiovascular Research Foundation (CRF), New York, New York, \\ United States
}

Thromb Haemost 2018;118:1343-1344.

The optimal management of venous thromboembolism (VTE) has long been a clinical challenge. ${ }^{1}$ Unprovoked VTE may be the earliest sign of cancer, and this association has been long recognized. ${ }^{2,3}$ Indeed, up to $10 \%$ of patients with unprovoked VTE will receive a diagnosis of cancer in the year after their diagnosis of VTE. ${ }^{4}$

Therefore, clinicians and scientists have long advocated systematic testing of asymptomatic individuals (i.e. screening) for pre-clinical occult malignancy. Subjecting patients to an extensive diagnostic workup could alter their clinical course: an earlier cancer diagnosis might potentially lead to earlier and more effective treatment and would also affect anticoagulation choice. However, routine screening for occult cancer after unprovoked VTE is not supported by current evidence. The Screening for Occult Malignancy in Patients with Idiopathic Venous Thromboembolism (SOME) trial randomized patients with unprovoked VTE to a limited screening strategy involving standard age- and sex-specific screening or to an extensive strategy that added computed tomography of the abdomen and pelvis. ${ }^{5}$ Among 854 patients, the primary outcome of the study (the number of cancers 'missed' at the initial screening but diagnosed by the end of the 1-year follow-up period) was $0.93 \%$ in the limited screening group and $1.18 \%$ in the extensive screening group.

Since a sub-group of high-risk patients could potentially benefit from a more extensive occult cancer screening strategy, investigators have developed risk scores that might provide a basis for effective screening and preventive strategies. For example, Jara-Palomares et al identified 6 independent predictors (RIETE score) assessed at the time of VTE presentation of occult cancer in a 24-month follow-up

received

June 19, 2018

accepted

June 19, 2018

Address for correspondence David Jiménez, MD, PhD, Respiratory and Medicine Department, Hospital Ramón y Cajal, Universidad de Alcalá (IRYCIS), 28034 Madrid, Spain (e-mail: djimenez.hrc@gmail.com).

period: male sex, age $>70$ years, chronic lung disease, anaemia (haemoglobin levels $<13 \mathrm{~g} / \mathrm{dL}$ for men and $<12 \mathrm{~g} / \mathrm{dL}$ for women), elevated platelet count $\left(\geq 350,000 \times 1,000 / \mathrm{mm}^{3}\right)$, prior VTE and recent surgery. ${ }^{6}$ For each patient, the score assigned 2 points each for the presence of age $>70$ years and anaemia, and 1 point each for the presence of male sex, chronic lung disease and raised platelet count; and 2 negative points for the presence of recent surgery. Patients with a total score of $\leq 2$ were assigned to the low-risk category, and those with a total score of $\geq 3$ points to the high-risk category. Six percent (95\% confidence interval $[\mathrm{CI}], 5.1-6.6 \%)$ of the low-risk patients versus $12 \%$ (95\% CI, 10.4-13.5\%) of the high-risk patients were diagnosed with cancer during follow-up. ${ }^{6}$ Ihaddadene et al performed a posthoc analysis of the SOME trial and found that age $\geq 60$ years (hazard ratio [HR], 3.1; $95 \% \mathrm{CI}$, $1.4-6.9 ; p=0.005$ ), previous provoked VTE ( $\mathrm{HR}, 3.2 ; 95 \% \mathrm{CI}$, $1.2-8.62 ; p=0.022$ ) and current smoker status (HR, 2.8; 95\% CI, $1.2-6.3 ; p=0.014)$ were associated with occult cancer detection (SOME score). ${ }^{7}$

To show that a prognostic model is valuable, it is not sufficient to show that it successfully predicts outcome in the initial development data. We need evidence that the model performs well for other groups of patients. ${ }^{8}$ It is important to check the proportion of patients classified by the rule in the different prognostic groups, as well as its accuracy and calibration. The validation cohort should represent an unselected group of patients with a wide spectrum of disease severity, and the predictors for the rule should be collected blinded from the final outcome.

In the previous issue of Thrombosis and Haemostasis, Kraaijpoel and a team of renowned clinical scientists

(c) 2018 Georg Thieme Verlag KG Stuttgart · New York
DOI https://doi.org/ $10.1055 / \mathrm{s}-0038-1667035$. ISSN 0340-6245. 
performed a posthoc analysis of the Hokusai-VTE trial to evaluate the performance of the RIETE and SOME scores for the occurrence of subsequent occult cancer in patients with acute VTE. $^{9}$ A total of 8,032 patients were included in the analysis. The incidence of occult cancer was $1.8 \%$ in patients with unprovoked VTE (5,359 patients, $67 \%$ ), and $2.1 \%$ in those with provoked VTE (2,673 patients, 33\%). The RIETE score classified $19 \%$ of patients as having a 'high risk' of occult cancer and the SOME score $16 \%$. In patients classified as 'high risk', the cumulative incidence of cancer diagnosis during follow-up was $2.9 \%$ (95\% CI, 2.1-3.9\%) for the RIETE score and $2.7 \%$ (95\% CI, 1.9-3.7\%) for the SOME score, corresponding to HRs of 1.8 (95\% $\mathrm{CI}, 1.3-2.5)$ and $1.5(95 \% \mathrm{CI}, 1.04-2.2)$, respectively. The $C-$ statistics of the RIETE and SOME scores were 0.62 (95\% CI, $0.57-0.66)$ and 0.59 (95\% CI, 0.55-0.62), respectively.

Various factors might explain the relatively poor predictive capability of the two models assessed in this study. The models' predictions might not be reproducible because of deficiencies in the modelling methods used in the study to derive the model. Poor performance could also arise from differences between the setting of patients in the new and derivation samples. Randomized controlled trials (RCTs) are currently the best approach to evaluate the effectiveness of therapies while accounting for the effects of unmeasured confounders and selection bias by indication. However, there is reasonable concern about inadequate representativeness of RCTs. In a recent study by the RIETE registry on the real-life use of direct oral anticoagulants in patients with VTE, 19\% met at least one exclusion criterion for the trials where the indication was established. ${ }^{10}$ The higher incidence of occult cancer in the sub-group of patients with provoked VTE (compared with the sub-group of patients with unprovoked VTE) might suggest that a non-representative group of patients were enrolled in the Hokusai-VTE trial.

Based on the results of the Kraaijpoel et al study, the value of these scores is questionable. Other factors such as presence of extensive VTE (including bilateral deep vein thrombosis) have recently shown promise, ${ }^{11}$ whereas more traditional cancer risk factors such as longstanding history of smoking, alcohol overuse, history of radiation and family history of early cancer may warrant further assessment. In addition, cancer-specific biomarkers might improve the discriminative performance of these risk scores and require further validation (ClinicalTrials. gov; NCT02739867). The final step would involve assessing the impact of its use on practice patterns, outcomes of care and costs. $^{12}$ Until these score are available, a limited screening strategy involving standard age- and sex-appropriate screening is advisable.

\section{Conflict of Interest}

None.

\section{References}

1 Schulman S, Ageno W, Konstantinides SV. Venous thromboembolism: past, present and future. Thromb Haemost 2017;117(07): 1219-1229

2 Ay C, Pabinger I, Cohen AT. Cancer-associated venous thromboembolism: burden, mechanisms, and management. Thromb Haemost 2017;117(02):219-230

3 Cohen AT, Katholing A, Rietbrock S, Bamber L, Martinez C. Epidemiology of first and recurrent venous thromboembolism in patients with active cancer. A population-based cohort study. Thromb Haemost 2017;117(01):57-65

4 Carrier M, Le Gal G, Wells PS, Fergusson D, Ramsay T, Rodger MA. Systematic review: the Trousseau syndrome revisited: should we screen extensively for cancer in patients with venous thromboembolism? Ann Intern Med 2008;149(05):323-333

5 Carrier M, Lazo-Langner A, Shivakumar S, et al; SOME Investigators. Screening for occult cancer in unprovoked venous thromboembolism. N Engl J Med 2015;373(08):697-704

6 Jara-Palomares L, Otero R, Jimenez D, et al; RIETE Investigators. Development of a risk prediction score for occult cancer in patients with VTE. Chest 2017;151(03):564-571

7 Ihaddadene R, Corsi DJ, Lazo-Langner A, et al. Risk factors predictive of occult cancer detection in patients with unprovoked venous thromboembolism. Blood 2016;127(16):2035-2037

8 Altman DG, Vergouwe Y, Royston P, Moons KG. Prognosis and prognostic research: validating a prognostic model. BMJ 2009; 338:b605

9 Kraaijpoel N, van Es N, Raskob GE, et al. Risk scores for occult cancer in patients with venous thromboembolism: a post hoc analysis of the Hokusai-VTE study. Thromb Haemost 2018;118 (07):1270-1278

10 Moustafa F, Pesavento R, di Micco P, et al; RIETE Investigators. Real-life use of anticoagulants in venous thromboembolism with a focus on patients with exclusion criteria for direct oral anticoagulants. Clin Pharmacol Ther 2018;103(04):684-691

11 Bikdeli B, Sharif-Kashani B, Bikdeli B, et al; RIETE Investigators. Impact of thrombus sidedness on presentation and outcomes of patients with proximal lower extremity deep vein thrombosis. Semin Thromb Hemost 2018;44(04):341-347

12 Moons KG, Royston P, Vergouwe Y, Grobbee DE, Altman DG. Prognosis and prognostic research: what, why, and how? BMJ 2009;338:b375 